\title{
Qp \\ 3-D Depth Migration via McClellan Transformations
}

\author{
by
}

\author{
Dave Hale
}

\begin{abstract}
DISCLAIMER
This report was prepared as an account of work sponsored by an agency of the United States Government. Neither the United States Government nor any agency thereof, nor any of their employees, makes any warranty, express or implied, or assumes any legal liability or responsibility for the accuracy, completeness, or usefulness of any information, apparatus, product, or process disclosed, or represents that its use would not infringe privately owned rights. Reference herein to any specific commercial product, process, or service by trade name, trademark, manufacturer, or otherwise does not necessarily constitute or imply its endorsement, recommendation, of favoring by the United States Government or any agency thereof. The views and opinions of authors expressed herein do not necessarily state or reflect those of the United States Government or any agency thereof.
\end{abstract}

Center for Wave Phenomena

Colorado School of Mines

Golden, Colorado 80401

Phone (303) 273-3557 


\title{
3-D depth migration via McClellan transformations
}

\author{
Dave Hale
}

\begin{abstract}
Three-dimensional seismic wavefields may be extrapolated in depth, one frequency at a time, by two-dimensional convolution with a circularly symmetric, frequency- and velocity-dependent filter. This depth extrapolation, performed for each frequency independently, lies at the heart of 3-D finite-difference depth migration. The computational efficiency of 3-D depth migration depends directly on the efficiency of this depth extrapolation.
\end{abstract}

McClellan transformations provide an efficient method for both designing and implementing two-dimensional digital filters that have a particular form of symmetry, such as the circularly symmetric depth extrapoiation filters used in 3-D depth migratinn. Given the coefficients of one-dimensional, frequency-and velocity-dependent filters used to accomplish 2-D depth migration, McClellan transformations lead to a simple and efficient algorithm for 3-D depth migration.

3-D depth migration via McClellan transformations is simple because the coefficients of two-dimensional depth extrapolation filters are never explicitly computed or stored; only the coefficients of the corresponding one-dimensional filter are required. The algorithm is computationally efficient because the cost of applying the two-dimensional extrapolation filter via McClellan transformations increases only linearly with the number of coefficients $N$ in the corresponding one-dimensional filter. This efficiency is not intuitively obvious, because the cost of convolution with a two-dimensional filter is generally proportional to $N^{2}$. Computational efficiency is particularly important for 3-D depth migration, for which long extrapolation filters (large $N$ ) may be required for accurate imaging of steep reflectors.

\section{INTRODUCTION}

The computational expense of 3-D poststack depth migration has motivated numerous papers describing different methods for performing this important step in 
seismic data processing. This paper describes yet another method, related to methods described by others, but with significant advantages in computational simplicity and efficiency.

The development of accurate 3-D poststack depth migration has been hindered historically by the fact that commonly used finite-diference migration methods cannot be easily extended from 2-D to 3-D. In particular, 2-D wepth migration methods that are based on implicit depth extrapolation of the seismic wavefield, such as the common "45-degree" finite-difference method (e.g., Claerbout, 1985), are difficult to extend to $3-\mathrm{D}$ processing.

Many authors, including Brown (1983), Yilmaz (1987, p. 404-405), and Kitchenside and Jakubowicz (1987), have noted the error in splitting the depth extrapolation process to alternately extrapolate along the inline $(x)$ and crossline $(y)$ directions, independently. Splitting is the most practical way to extend implicit extrapolation methods from 2-D to 3-D, but as these authors have shown, the errors in splitting depend significantly on reflector dip and azimuth. Specifically, for reflector dips greater than about 20 degrees and reflector azimuths near 45 degrees, measured with respect to either the $x$ or $y$ directions, splitting yields unacceptable errors in reflector positioning. Although techniques for reducing the error in splitting have been demononstrated (Ristow, 1980; Graves and Clayton, 1990), these techniques significantly increase both the computational cost and complexity of implicit depth extrapolation methods.

$S$ ume migration methods developed more recently, such as reverse-time migration, may be extended from 2-D to 3-D relatively easily, as demonstrated by Chang and McMechan (1989). Reverse-time migration, because it is based on an explicit backwards extrapolation in time, requires no splitting. However, several authors (Reshef and Kessler, 1989; Blacquière et al., 1989) have noted that 3-D reverse-time migration is relatively expensive, due to the large number of computations and the large amounts of computer memory required by this method.

Reshef and Kessler (1989) discuss the practical aspects of extending a generalized phase-shift method for depth migration (Kosloff and Kessler, 1987) from 2-D to 3-D. Because this method is based on explicit depth extrapolation via a two-way wave equation, splitting is unnecessary in their 3-D implementation. However, they note two difficulties with this method that stem from the use of a two-way wave equation. First, as noted by Kosloff and Kessler (1987), the method requires the attenuation of exponentially growing evanescent energy via a spatially varying filter. Second, the method uses impedance matching (Baysal et al., 1984) to attenuate unwanted reflections, but this impedance matching fails for waves propagating obliquely to a vertical velocity gradient. Both exponentially growing evanescent energy and unwanted reflections are symptoms of depth extrapolation with a two-way wave equation.

As noted by Claerbout (1985, p. 55), the problems associated with a two-way wave equation can be avoided by using a one-way wave equation for depth extrapolation of the seismic wavefield. However, depth migration via the one-way wave equation has traditionally been accomplished via an implicit extrapolation method (as in 45-degree finite-difference migration), which requires splitting in its extension from 2-D to 3-D. 
Splitting is unnecessary for explicit extrapolation methods. Holberg (1988) describes a method for designing explicit extrapolation filters for the one-way wave equation, and Blacquière et al. (1989) have extended this method for use in 3-D depth migration. Specifically, Blacquière et al. compute and tabulate the coefficients of two-dimensional extrapolation filters. Each of these filters corresponds to a particular ratio of frequency to velocity. Depth extrapolation is then performed for each frequency, independently, by convolving a two-dimensional extrapolation filter with the seisnuic wavefield. Lateral velocity variations are handled by leciing the coefficients of the extrapolation filter (extracted from the table) vary as velocity varies in both the $x$ and $y$ directions.

While direct convolution with two-dimensional extrapolation filters is more accurate than splitting, it is also computationally expensive. The cost of direct convolution is proportional to $N^{2}$, where $N$ is the number of coefficients in the corresponding onedimensional extrapolation filter that one might use in performing 2-D depth migration. In contrast, the cost of the splitting method is proportional to $N$. The relatively high cost of direct convolution with two-dimensional extrapolation filters motivated Kitchenside and Jakubowicz (1987) and Kitchenside (1988) to investigate alternative extrapolation schemes that combine direct convolution, splitting, and Fourier transform methods to achieve computational efficiency.

The 3-D depth migration method described in this paper is similar to that derribed by Blacquière et al. (1989), but with two significant advantages:

- The coefficients of two-dimensional depth extrapolation filters are never computed or tabulated. Only the coefficients of the corresponding one-dimensional extrapolation filters (identical to those used in $2-\mathrm{D}$ depth migration) are required.

- The cost of depth extrapolation increases only lincarly with $N$, the number of coefficientis in the one-dimensional exträpolation filters.

The computational simplicity and efficiency of this method stem from the design and implementation of depth extrapolation. filters via McClellan transformations.

\section{DEPTH EXTRAPOLATION FOR 2-D AND 3-D MIGRATION}

2-D migration, when performed by recursive extrapolation of the seismic wavefield in depth, requires an extrapolation filter with a Fourier transform that approximates

$$
D(k) \equiv e^{i \frac{\Delta x}{\Delta x}\left[\left(\frac{w \Delta x}{v}\right)^{2}-k^{2}\right]^{1 / 2}},
$$

where $\omega$ denotes freq $\_n r y$ (in radians per unit time), $v$ denotes velocity, and $\Delta z$ and $\Delta x$ denote vertical and horizontal spatial sampling intervals, respectively. Wavenumber $k$ (measured in radians per sample in the $x$ direction) is normalized such that any 
distance quantity is measured in terms of the number of horizontal sampling intervals $\Delta x$. With this normalization, two dimension-less constants, $\Delta z / \Delta x$ and $\omega \Delta x / v$, uniquely specify the desired transform $D(k)$.

The desired transform $D(k)$ defined by equation (1) is appropriate for waves traveling one way, either down or up. In depth extrapolation of CMP stacked data, which corresponds to waves propagating both down and up, one may use the "exploding reflectors" concept and replace velocity $v$ with half-velocity $v / 2$ (e.g., Claerbout, 1985).

The explicit depth extrapolation filters of interest here consist of $N$ complex coefficients $h_{n}$, with the Fourier transform

$$
H(k) \equiv \sum_{n=\frac{-N+1}{2}}^{\frac{N-1}{2}} h_{1} e^{-i k n} \approx D(k)
$$

The symmetry of $D(k)$ with respect to $k$ implies that the complex extrapolation filter coefficients $h_{n}$ are even; that is, $h_{-n}=h_{n}$. Therefore, the number of coefficients $N$ should be odd. The number of unique coefficients $h_{n}$ is $N_{h} \equiv(N+1) / 2$, and the Fourier transform $H(k)$ inay be expressed as

$$
H(k)=h_{0}+2 \sum_{n=1}^{N_{h}-1} h_{n} \cos (k n) .
$$

Methods for computing the $N_{h}$ coefficients $h_{n}$ to obtain a satisfactory approximation $H(k)$ to the desired transform $D(k)$ are described by Holberg (1988) and Hale (1990).

In 2-D migration, extrapolation in depth may be accomplished, for each frequency $\omega$ independently, by convolving with the one-diniensional extrapolation filter $h_{n}$. For 3-D migration, depth extrapolation may be performed by convolution with a circularly symmetric two-dimensional filter. This filter should have a Fourier transform approximating that specified in equation (1), but with wavenumber $k$ replaced by $k=\sqrt{k_{x}^{2}+k_{y}^{2}}$, where $k_{x}$ and $k_{y}$ denote inline and crossline wavenumbers, respectively, both normalized by the inline sampling interval $\Delta x$, which I assume is equal to the crossline sampling interval $\Delta y$. The desired Fourier transform of the two-dimensional extrapolation filter for 3-D migration is then

$$
D\left(k_{x}, k_{y}\right)=e^{i \frac{\Delta x}{\Delta x}\left[\left(\frac{\omega \Delta x}{v}\right)^{2}-k_{x}^{2}-k_{y}^{2}\right]^{1 / 2}} .
$$

Splitting in 3-D migration is based on the approximation (e.g., Brown, 1983)

$$
D\left(k_{x}, k_{y}\right) \approx e^{i \frac{\Delta_{z}}{\Delta x}\left\{\left[\left(\frac{\omega \Delta x}{v}\right)^{2}-k_{x}^{2}\right]^{1 / 2}+\left[\left(\frac{\omega \Delta x}{v}\right)^{2}-k_{y}^{2}\right]^{1 / 2}-\frac{\omega \Delta x}{v}\right\} .}
$$

By separating the $k_{x}$ and $k_{y}$ terms in the exponent of equation (4), we approximate the two-dimensional convolution implied by equation (3) as a relatively inexpensive cascade of one-dimensional convolutions in the $x$ direction, followed by one-dimensional 
convolutions in the $y$ direction. Comparing equations (3) and (4), the splitting approximation is best for $v k_{y} / \omega \approx 0$ or $v k_{x} / \omega \approx 0$, corresponding to reflectors dipping in the $x$ or $y$ directions. Splitting yields large errors for $v k_{x} / \omega \approx v k_{y} / \omega \gg 0$, corresponding to steep reflectors dipping at 45 degrees azimuth between the $x$ and $y$ directions.

\section{MCCLELLAN TRANSFORMATIONS}

Using McClellan transformations, one can avoid the errors in splitting, without computing the coefficients of two-dimensional extrapolation filters. As will be shown below, we need only the one-dimensional filter coefficients $h_{n}$ used in 2-D migration.

McClellan transformations (McClellan, 1973; Mersereau et al., 1976) are based on the following trigonometric identity

$$
\cos (n \theta)=2 \cos (\theta) \cos [(n-1) \theta]-\cos [(n-2) \theta]
$$

which is a special case of the recursive formula for the Chebyshev polynomials $T_{n}(x)$ defined by $T_{n}(\cos \theta) \equiv \cos (n \theta)$ (e.g., Abramowitz, M. and Stegun, I. A., 1965, p. $776)$. With this identity, each of the $\cos (k n)$ terms in equation (2) may be computed recursively from $\cos k$ :

$$
H(k)=h_{0}+2 h_{1} \cos k+2 h_{2}(2 \cos k \cos k-1)+\ldots
$$

Recognizing that the filter $H(k)$ may be written entirely in terms of $h_{n}$ and $\cos k$, McClellan and Chan (1977) noted that convolution with the filter $h_{n}$ may be accomplished via the recursive Chebyshev filter structure illustrated in Figure 1.

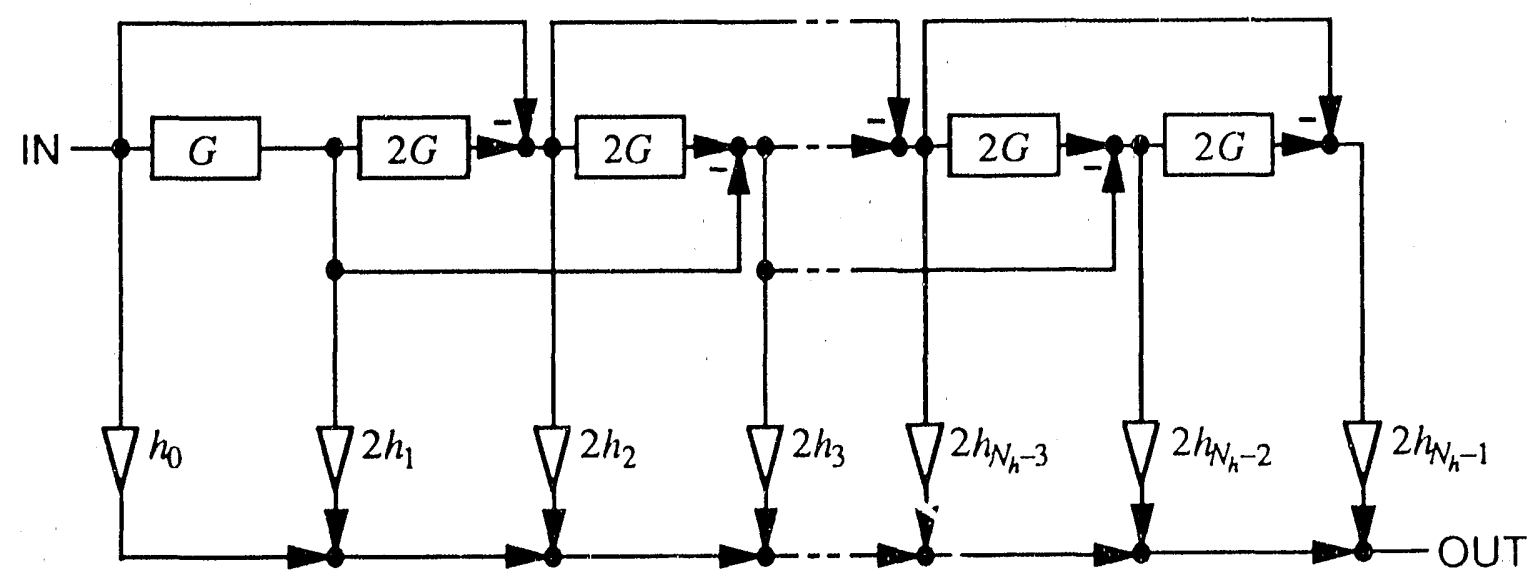

FIG. 1. Chebyshev filter structure for convolution with the filter $h_{n}$. The upper part of this structure implements the Chebyshev "ecursion for $\cos (k n)$ in terms of $G(k)=\cos k$.

For one-dimensional filters, $G(k)=\cos (k)$ in Figure 1 is the Fourier transform of the filter $g_{j}$ defined by

$$
g_{j} \equiv \begin{cases}1 / 2, & \text { if } j= \pm 1 \\ 0, & \text { otherwise }\end{cases}
$$


which has only two non-zero coefficients. This Chebyshev filter structure is, however, cumbersome for one-dimensional filters. Direct convolution with the coefficients $h_{n}$ is both simpler and more efficient than the structure illustrated in Figure 1.

The Chebyshev filter structure is most advantageous for two-dinensional filters with quadrantal symmetry, such as circularly symmetric two-dimensional extrapolation filters, for which $k=\sqrt{k_{x}^{2}+k_{y}^{2}}$. Such filters may be implemented by the Chebyshev filter structure by simply replacing $G$ in Figure 1 with $G\left(k_{x}, k_{y}\right)=\cos \sqrt{k_{x}^{2}+k_{y}^{2}}$.

An exact representation of $G\left(k_{x}, k_{y}\right)$ in the Chebyshev filter structure of Figure 1 would result in a computational cost greater than that of direc: two-dimensional convolution. Therefore, McClellan (1973) suggested the approximation

$$
G\left(k_{x}, k_{y}\right)=-1+\frac{1}{2}\left(1+\cos k_{x}\right)\left(1+\cos k_{y}\right) \text {. }
$$

This approximation is the Fourier transform of the compact two-dimensional filter illustrated in Figure 2. Replacing the ccefficients of the one-dimensional filter $g_{j}$ defined by equation (5) with the two-dimensional filter of Figure 2 transforms any one-dimensional filter to a two-dimensional filter with approximate circular symmetry. Note that the Chebyshev filter structure is unchanged, that the filter coefficients $h_{n}$ are the coefficients of the original one-dimensional filter, and that the cost of applying the two-dimensional filter grows linearly with the number of filter coefficients $N_{h}$.

\begin{tabular}{|c|c|c|}
\hline$\frac{1}{8}$ & $\frac{1}{4}$ & $\frac{1}{8}$ \\
\hline$\frac{1}{4}$ & $-\frac{1}{2}$ & $\frac{1}{4}$ \\
\hline$\frac{1}{8}$ & $\frac{1}{4}$ & $\frac{1}{8}$ \\
\hline
\end{tabular}

FIG. 2. Original McClellan transformation filter - transforms any one-dimensional filter into an approximately circularly symmetric two-dimensional filter, implemented via the Chebyshev recursion in Figure 1.

The coefficients of the McClellan transformation filter illustrated in Figure 2 are independent of the coefficients of the depth extrapolation filter. Therefore, that part of the Chebyshev filter structure that performs convolution with the transformation filter (the upper part of Figure 1) can be optimized independently from the part that multiplies the seismic wavefield by the coefficients $h_{n}$ of the extrapolation filter.

Figure 3 shows contours of constant amplitude and phase (constant $k$ ) for any two-dimensional filter designed and implemented via the McClellan transformation of equation (6) and Figure 2. As illustrated in Figure 3, this transformation is best for small $k_{x}$ and $k_{y}$. The transformation is exact for $k_{x}=0$ or $k_{y}=0$, but exhibits increasing error with increasing $k$ for $k_{x} \approx k_{y}$. 


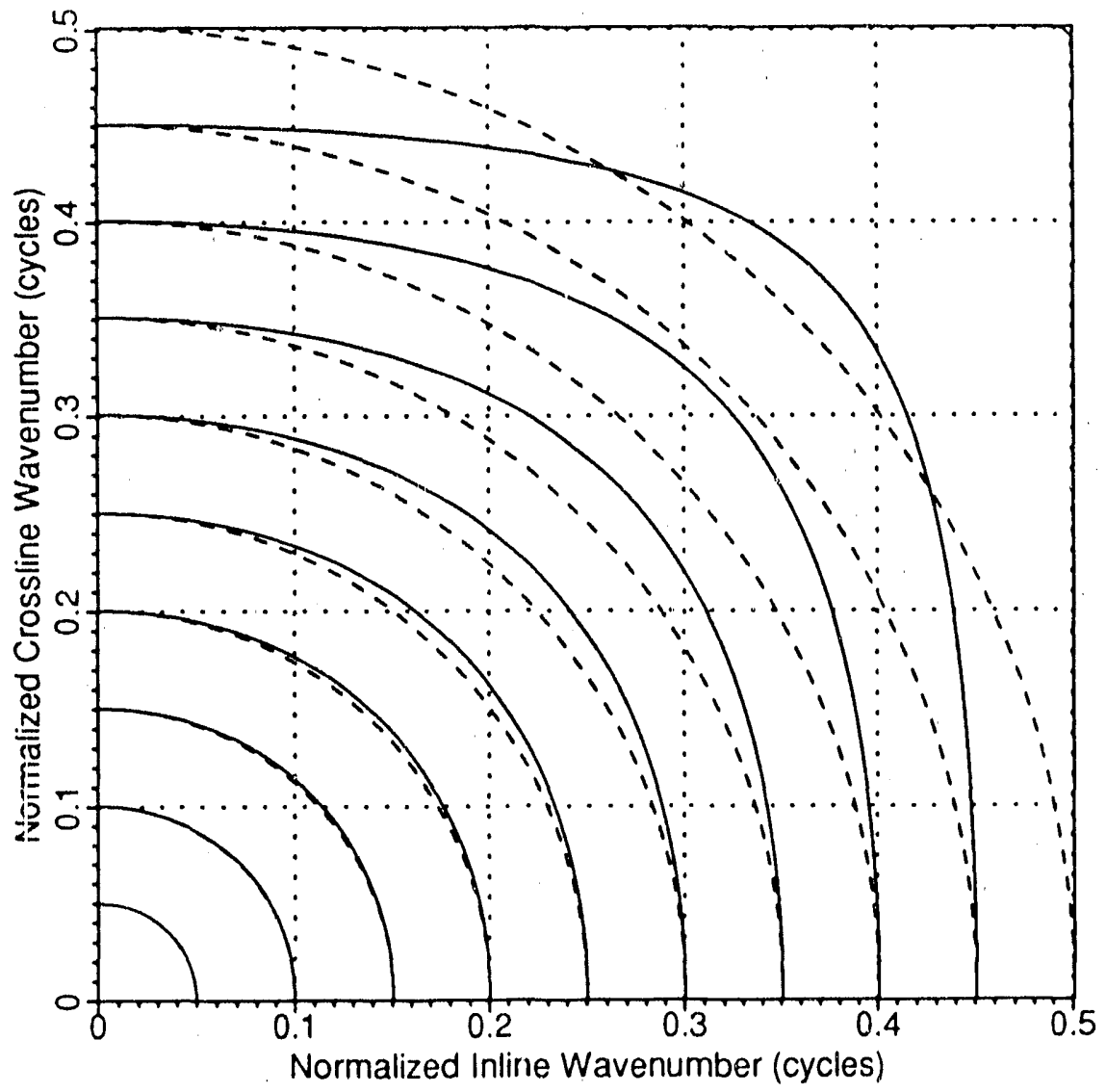

Fig. 3. Contours of constant amplitude and phase (constant $k$ ) for two-dimensional filters designed and implemented via the original McClellan transformation of Figure 2. Contours of ideal circularly symmetric filters are plotted with dashed lines. Compare with Figure 5 . In this figure, normalized wavenumbers $k_{x}$ and $k_{y}$ are measured in cycles per spatial sampling interval. Thus, $k=0.25$ is one-half the Nyquist wavenumber. 
Figure 4 shows the coefficients of an improved McClellan transformation filter, which has the Fourier transform

$$
\begin{aligned}
G\left(k_{x}, k_{y}\right)= & -1+\frac{1}{2}\left(1+\cos k_{x}\right)\left(1+\cos k_{y}\right) \\
& -\frac{c}{2}\left(1-\cos 2 k_{x}\right)\left(1-\cos 2 k_{y}\right) .
\end{aligned}
$$

Contours of constant $k$ for this filter are plotted in Figure 5. The constant $c$ in equation (7) was determined by matching exactly a particular $k$ along the diagonal $k_{x}=k_{y}$. In this case, the value $k=\pi / 3$ was chosen to determine the constant $c \approx 0.3255$. Further improvement is possible, by using larger transformation filters. For a thorough discussion on the design of McClellan transformations, see Mersereau et al. (1976).

\begin{tabular}{|c|c|c|c|c|}
\hline$-\frac{c}{8}$ & 0 & $\frac{c}{4}$ & 0 & $-\frac{c}{8}$ \\
\hline 0 & $\frac{1}{8}$ & $\frac{1}{4}$ & $\frac{1}{8}$ & 0 \\
\hline$\frac{c}{4}$ & $\frac{1}{4}$ & $-\frac{1+c}{2}$ & $\frac{1}{4}$ & $\frac{c}{4}$ \\
\hline 0 & $\frac{1}{8}$ & $\frac{1}{4}$ & $\frac{1}{8}$ & 0 \\
\hline$-\frac{c}{8}$ & 0 & $\frac{c}{4}$ & 0 & $-\frac{c}{8}$ \\
\hline
\end{tabular}

Frg. 4. Improved McClellan transformation filter for a circularly symmetric twodimensional filter implemented via the Chebyshev recursion in Figure 1. The constant $c \approx 0.0255$ yields a two-dimensional filter that is more nearly circularly symmetric than the original McClellan transformation. Compare this transformation filter with that in Figure 2.

The error in McClellan transformations, unlike the error in splitting, depends only on the magnitude of inline and crossline wavenumbers $k_{x}$ and $k_{y}$; this error is independent of frequency $\omega$. For example, Figure 5 suggests that the improved McClellan transformation is adequate for all wavenumbers less than half-Nyquist. In any case, neither the original nor the improved McClellan transformation yields an exactly circularly symmetric extrapolation filter. In the next section, I compare the accuracy of 3-D migration with the original and improved McClellan transformations with the accuracy obtained by splitting.

\section{3-D MIGRATION IMPULSE RESPONSES}

Following closely the work of Blacquière et al. (1989), I developed a 3-D depth migration method based on a table of extrapolation filters, one filter for each ratio 


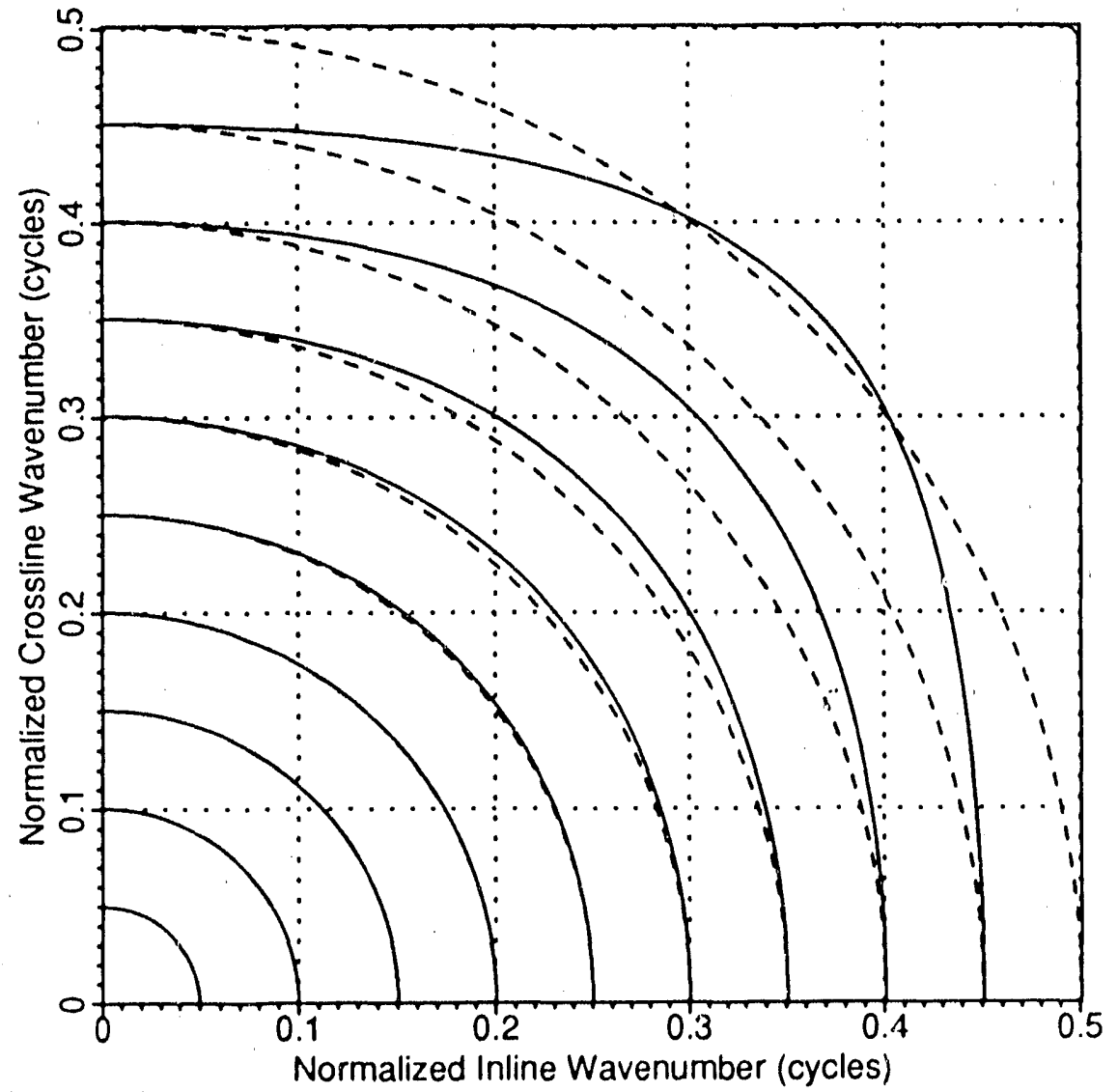

FIG. 5. Contours of constant amplitude and phase (constant $k$ ) for two-rimensional filters designed and implemented via the improved McClellan transformation filter of Figure 4. Contours of ideal circularly symmetric filters are plotted with dashed lines. Compare with Figure 3. In this figure, normalized wavenumbers $k_{x}$ and $k_{y}$ are measured in cycles per spatial sampling interval. Thus, $k=0.25$ is one-half the Nyquist wavenumber. 
of $\omega \Delta x / v$ tabulaiad. This table is the same table of one-dimensional filters that I use in 2-D depth migration. Specifically, I computed and tabulated stable explicit extrapolation filters via the modified Taylor series method described by Hale (1990). In practice, one-dimensional extrapolation filters designed using any method, such as that described by Holberg (1988), can be transformed into two-dimensional filters by the McClellan transformations described in the previous section.

Because only the coefficients $h_{n}$ of one-dimensional filters must be tabulated, I can afford to sample $\omega \Delta x / v$ in equation (1) finely, thereby avoiding the need to interpolate filter coefficients for a particular $\omega \Delta x / v-$ I simply choose the coefficients corresponding to the nearest value of $\omega \Delta x / v$ tabulated. The extrapolation filters are then applied via the Chebyshev filter structure of Figure 1, which permits the extrapolation filter coefficients $h_{n}$ to vary as velocity $v=v(x, y)$ varies laterally with $x$ and $y$. One can readily verify that doing so is equivalent to letting the coefficients of a two-dimensional extrapolation filter vary with output location, as done by Blacquière et al. (1989).

Because the ability of such a "table-driven" 3-D migration to handle lateral velocity variations has been well demonstrated by Blacquière et al. (1989), the tests described below were performed with constant velocity. These tests serve merely to demonstrate the implementation of the McClellan transformations described in the previous section.

The impulse responses of three different 3-D migration methods are illustrated in Figures 6 through 11. The three methods tested were based on depth extrapolation via

- splitting according to equation (4).

- the original McClellan transformation illustrated in Figures 2 and 3.

- the improved McClellan transformation illustrated in Figures 4 and 5.

In all of these tests, the spatial sampling intervals $\Delta x=\Delta y=\Delta z=10 \mathrm{~m}$, the time sampling interval $\Delta t=10 \mathrm{~ms}$, and the velocity $v=2 \mathrm{~km} / \mathrm{s}$. The input trace located at $x=y=0$ contained a single, zero-phase wavclet centered at $t=0.46 \mathrm{~s}$, with frequencies between 0 and $30 \mathrm{~Hz}$.

Figures 6,7 , and 8 illustrate 3-D migration impulse responses for the crossline coordinate $y=0$. These migration impulse responses for $y=0$ are dominated by wavenumber $k_{y}=0$, for which splitting and either McClellan transformation are expected to be accurate. Therefore, these particular inline views of the impulse responses are quite similar.

Figures 9,10 , and 11 illustrate 3-D migration impulse responses for the depth $z=250 \mathrm{~m}$. As illustrated in Figures 6, 7, and 8, this depth corresponds to a reflector dip of about 60 degrees. The error in splitting is clearly illustrated in Figure 9. Figure 9 is comparable to Figure 6-27 of Yilmaz (1987, p. 405), which further illustrates the azimuth-dependent errors in splitting. The original McClellan transformation 


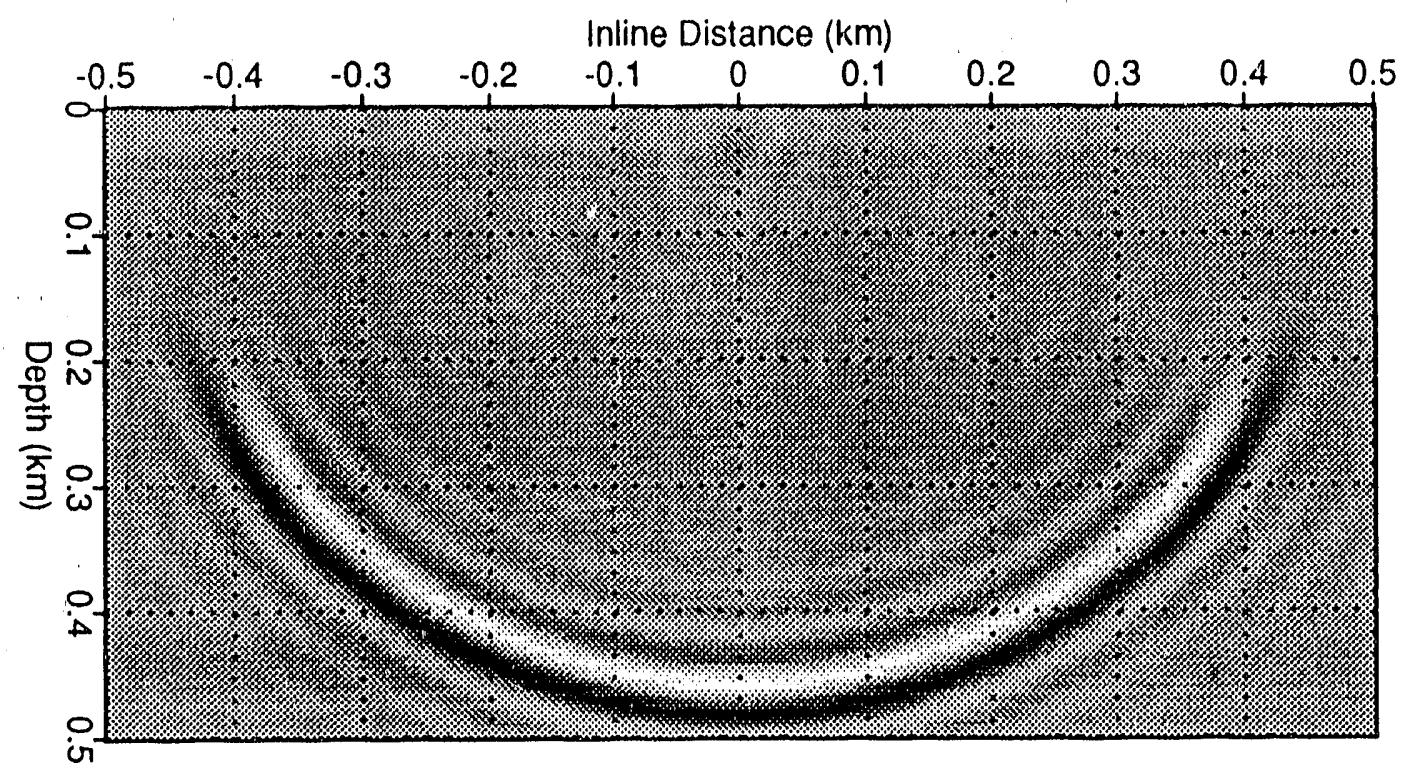

Fig. 6. Impulse response of 3-D migration via splitting, for the crossline coordinate $y=0$. Compare with Figures 7 and 8 .

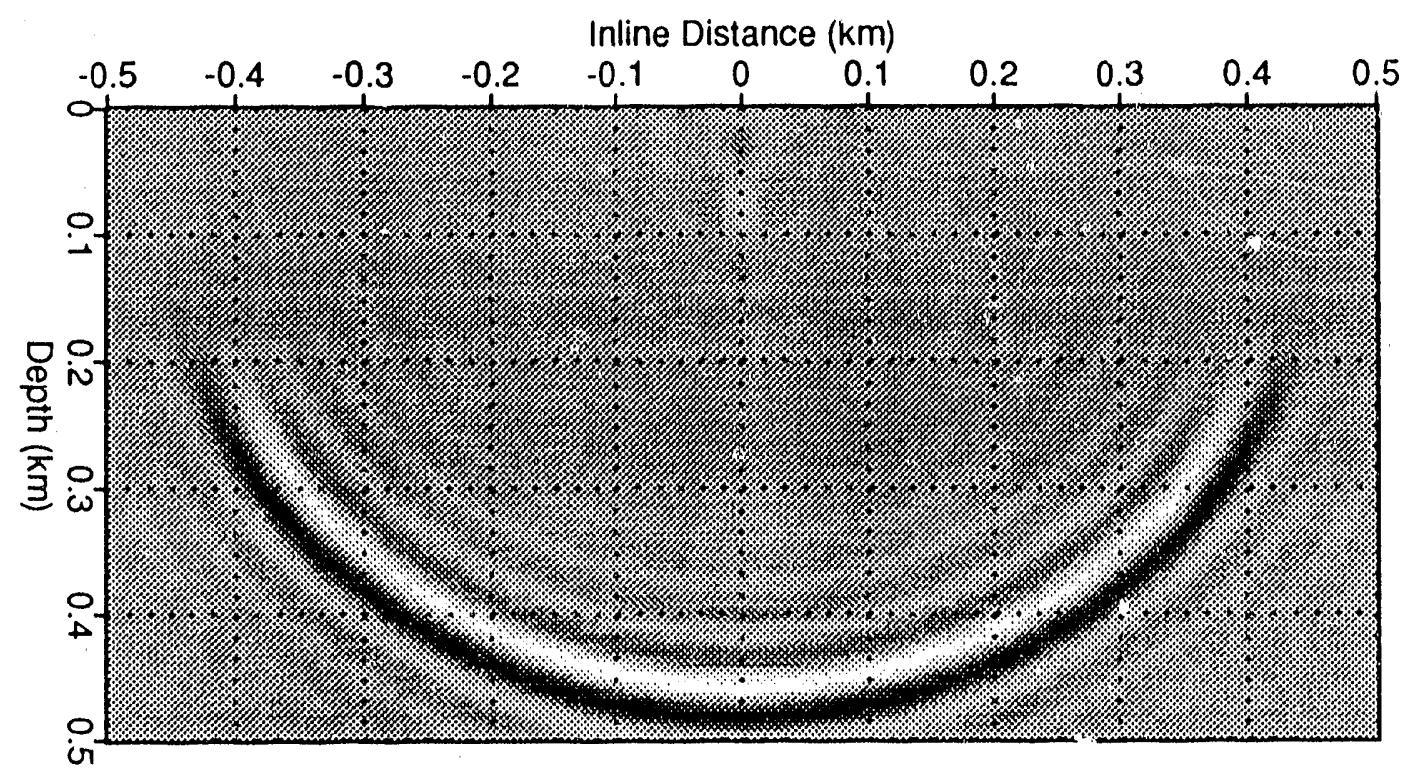

FIG. 7. Impulse response of 3-D migration via the original McClellan transformation, for the crossline coordinate $y=0$. Compare with Figures 6 and 8 . 


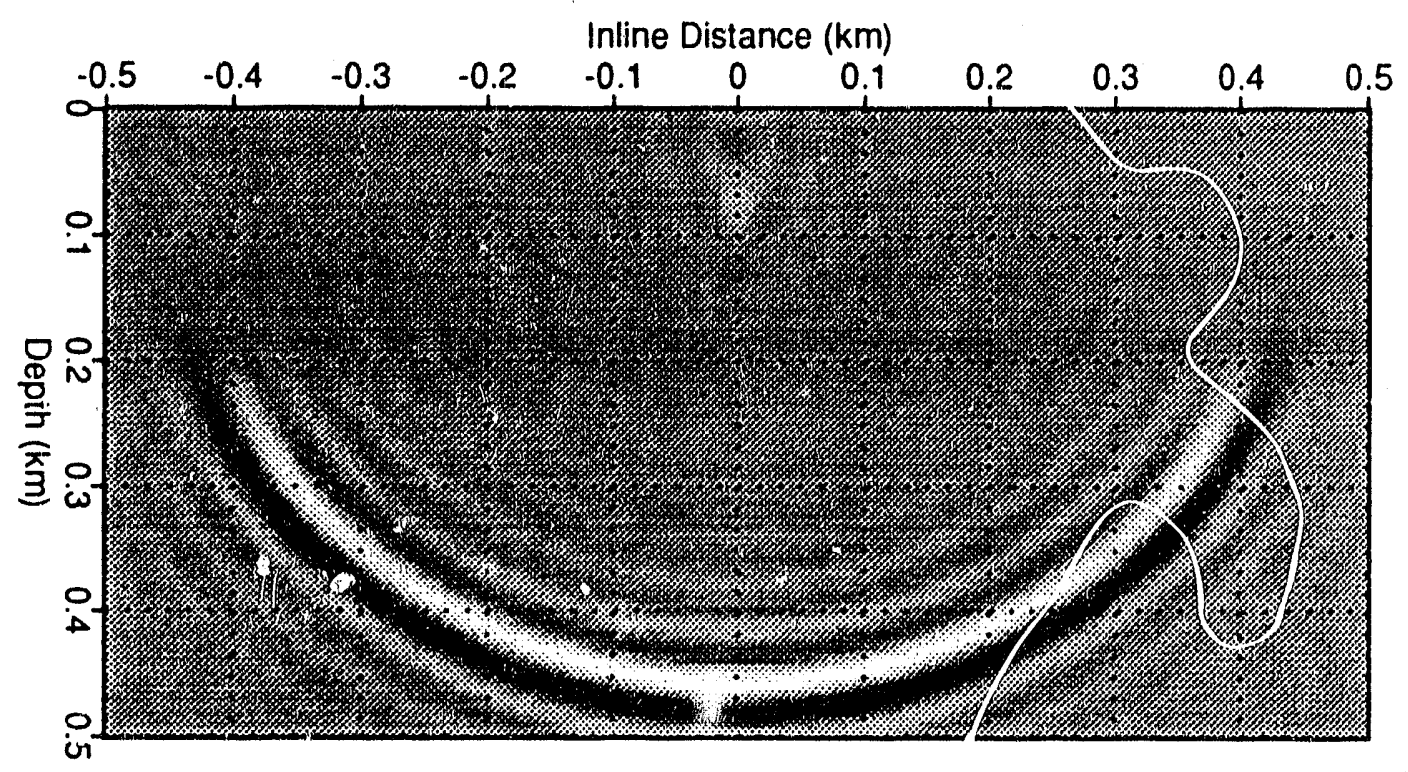

FIG. 8. Impulse response of 3-D migration via the improved McClellan transformation, for the crossline coordinate $y=0$. Compare with Figures 6 and 7 .

yields the nearly circular response seen in Figure 10, with only a slight distortion evident. The improved McClellan transformation yields tise apparently circular response exhibited in Figure 11.

Figures 9,10 , and 11 have all been plotted with the same amplitude scaling. The impulse response of $3-D$ migration via the splititing method (Figure 9) has measurably higher amplitude than either of the impulse responses obtained via McClellan transformaticil. Although the details are omitted here, a stationary phase estimate of the amplitude of the impulse response for splitting via equation (4) predicts that the amplitude in Figure 9 is $40 \%$ higher than the amplitude predicted by the correct equation (3). This difference of $40 \%$ accounts for the observed difference in amplitude between Figure 9 and Figures 10 and 11. In other words, 3-D migration via the splitting method may yield erroneously high reflector amplitudes, in addition to the more obvious errors in reflector positioning.

\section{COMPUTATIONAL EFFICIENCY}

McClellan transformations were first described in Geophysics by McClellan and Parks (1972) in a paper that showed how transformations could be used to design dip filters. At that time, the goal of the transformation method was merely to reduce the computation required to design two-dimensional filters. Later, several authors (MecklenBräuker and Mersereau, 1976; McClellan and Chan, 1977) noted that the transformation method could also significantly reduce the number of arithmetic operations required to implement two-dimensional filters, when compared with either direct convolution or fast Fourier tranform (FFT) implementations. 


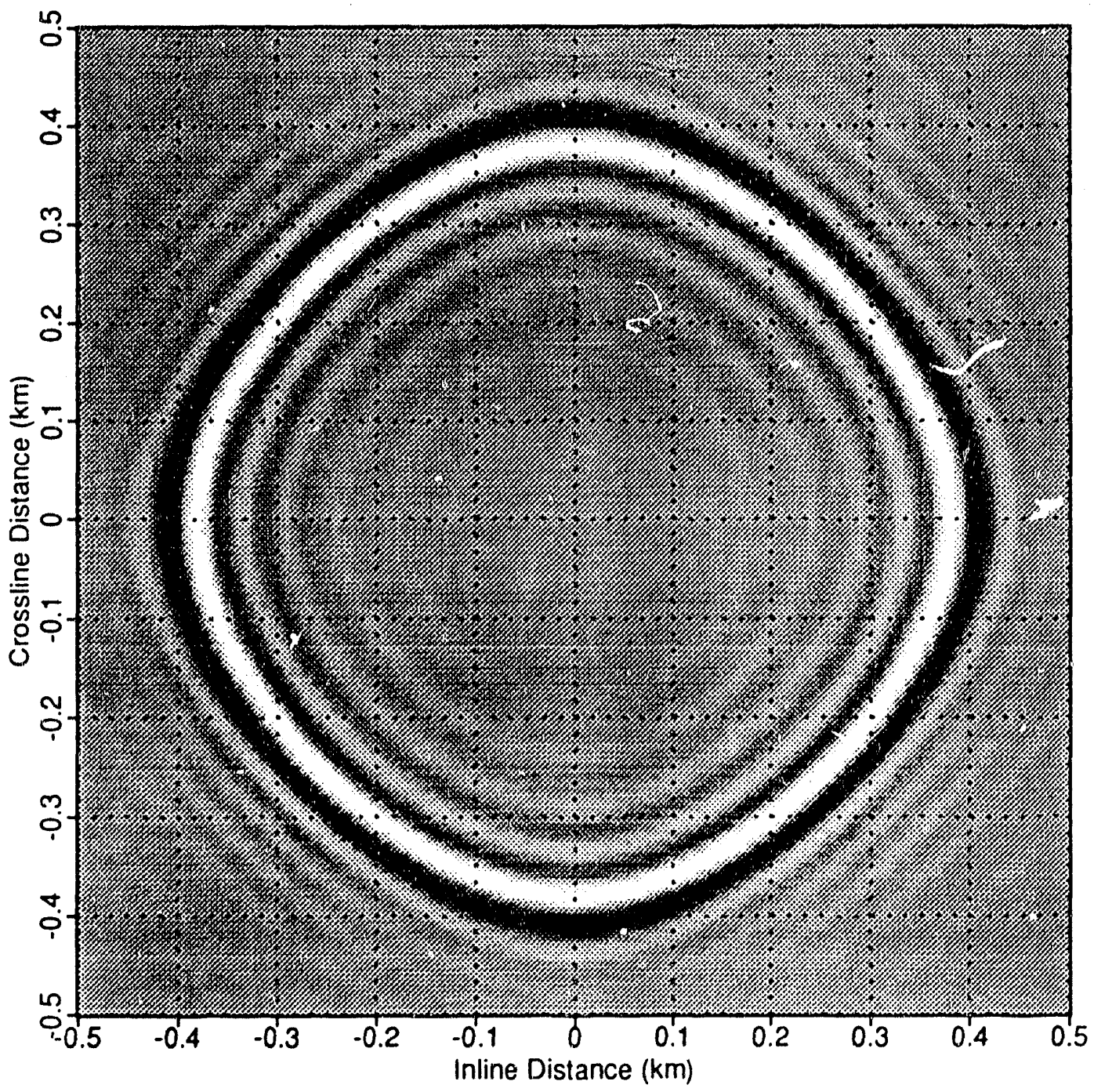

FIG. 9. Impulse response of 3-D migration via splitting, for the depth $z=250 \mathrm{~m}$. Compare with Figures 10 and 11. 


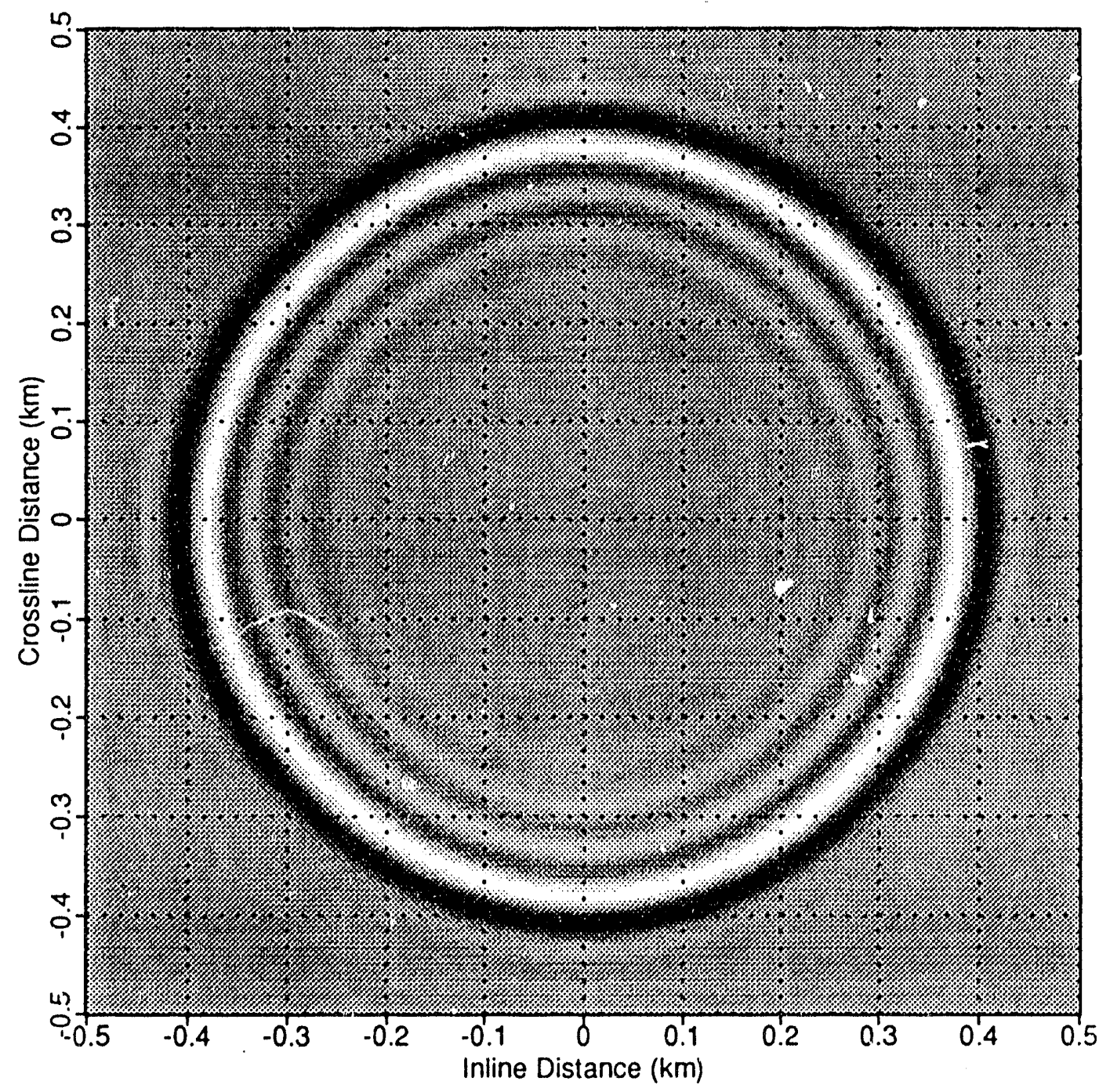

FIG. 10. Impulse response of 3-D migration via the original McClellan transformation, for the depth $z=250 \mathrm{~m}$. Compare with Figures 9 and 11 . 


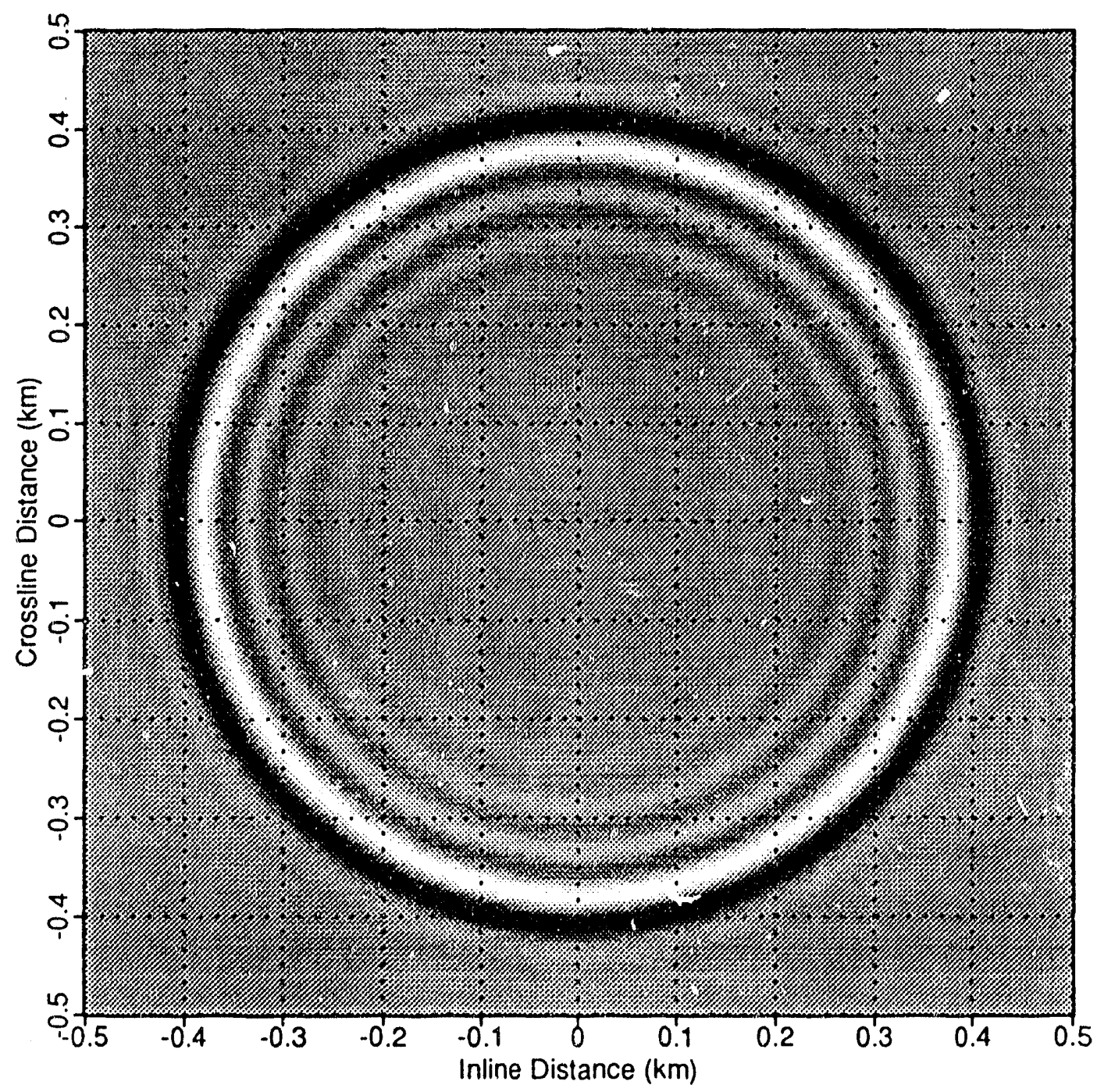

Fig. 11. Impulse response of 3-D migration via the improved McClellan transformation, for the depth $z=250 \mathrm{~m}$. Compare with Figures 9 and 10 . 
A typical application of McClellan transformations in signal processing has been the design and implementation of circularly symmetric low-pass filtcrs (e.g., Hazra and Reddy, 1986). The coefficients of such filters are typically real and constant, so that FFT implementations are a feasible alternative. McClellan transformation of depth extrapolation filters for depth migration is particularly attractive because (1) these filters have complex coefficients and (2) these coefficients may vary spatially. Recalling that a single complex multiplication requires six floating point operations (4 multiplications and 2 additions), note that only $N_{h}$ complex multiplications per output sample are required by the Chebyshev filter structure illustrated in Figure 1. Furthermore, unlike an FFT implementation, the Chebyshev filter structure permits the filter coefficients $h_{n}$ to vary as velocity varies with $x$ and $y$, as required for depth migration.

The number of floating point operations (FLOPS) per output sample required by four different depth extrapolation filters are plotted in Figure 12. The four methods for which costs are shown are splitting, the original McClellan transformation, the improved McClellan transformation, and direct convolutiun. For each of these methods, the number of (FLOPS) per output sample is plotted for $N_{h}=10,20$, and 30 coefficients. Although either McClellan transformation method requires more FLOPS than the splitting method to achieve the higher accuracy exhibited in Figures 10 and 11 , the cost of these transformation methods grows linearly with the number of filter coefficients $N_{h}$. In contrast, the cost of direct convolution grows quadratically as $N_{h}^{2}$. In practice, $3-\mathrm{D}$ depth migration via McClellan transformations will be substantially more efficient than direct convolution.

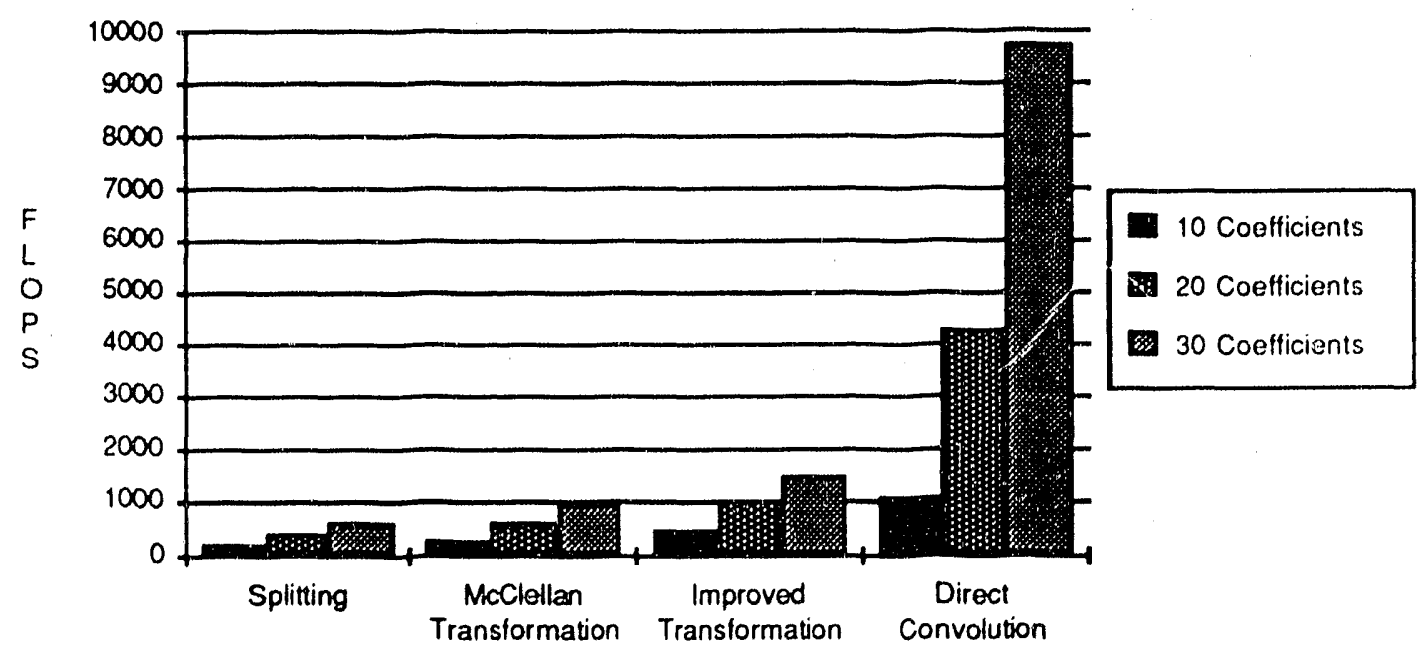

FIG. 12. Number of floating point operations (FLOPS) per output sample required by four different depth extrapolation methods. For each method, the number of FLOPS required is plotted for $N_{h}=10,20$, and 30 coefficients.

The number of FLOPS required for direct convolution, as plotted in Figure 12, was computed by exploiting the circular symmetry of two-dimensional extrapolation filters. Specifically, only $N_{h}\left(N_{h}+1\right) / 2$ complex multiplications are required for direct convolution; this savings is reflected in Figure 12. 


\section{CONCLUSIONS}

Given the advantages of performing 2-D depth migration via a table of explicit depth extrapolation filters, McClellan transformations extend these advantages from 2-D to 3-D depth migration. In this context of explicit depth extrapolation filters, McClellan transformations offer significant features:

- Only the coefficients of one-dimensional extrapolation filters, the same filters used in 2-D depth migration, need be tablilated. This simplifies the design of two-dimensional extrapolation filters for 3-D depth migration, while significantly reducing the amount of computer memory required to hold the tabulated filter coefficients.

- McClellan transformations are more efficient, requiring fewer floating point operations, than direct convolution with a two-dimensional extrapolation filter. In particular, the cost of 3-D depth migration grows only linearly with the length of the tabulated one-dimensional extrapolation filters.

- Even the simplest McClellan trausformation yields significantly gieater accuracy than does the splitting method typically used in implicit schemes for 3-D depth migration, for only slightly greater computational cost. The accuracy and computational cost of 3-D depth migration via McClellan transformations depends on the number of coefficients in the transformation filter. More accurate (and, thus, more costly) McClellan transformations may be designed and implemented as necessary.

The computational simplicity and efficiency of McClellan transformations make them an attractive method for implementing 3-D depth migration.

\section{ACKNOWLEDGMENT'S}

Thanks to Justin Hedley for help in searching the signal processing literature for references to McClellan transformations. I also thank Ken Larner for his critical reading of the first draft of this paper. Financial support for this work was provided in part by the United States Department of Energy, Grant Number DE-FG02-89ER14079. (This support does not constitute an endorsement by DOE of the views expressed in this paper.) Support was also provided by the members of the Consortium Project on Seismic Inverse Methods for Complex Structures at the Center for Wave Phenomena, Colorado School of Mines.

\section{REFERENCES}

Abramowitz, M., and Stegun, I. A., 1965, Handbook of mathematical functions: Dover Publications, Inc.

Baysal, E., Kosloff, D. D., and Sherwood, J. W. C., 1984, A two-way nonreflecting wave equation: Geophysics, 49, 132-141. 
Biacquière, G., Debeye, H. W. J., Wapenaar, C. P. A., and Berkhout, A. J., 1989, 3D table-driven migration: Geophys. Prosp., 37, 925-958.

Brown, D. L., 1983, Applications of operator separation in reflection seismology: Geophysics, 48, 288-294.

Chang, W., and McMechan, G. A., 1989, 3D acoustic reverse-time migration: Geophys. Prosp., 37, 243-256.

Claerbout, J. F., 1985, Imaging the earth's interior: Blackwell Scientific Publications.

Graves, R. W., and Clayton, R. W., 1990, Modeling acoustic waves with paraxial extrapolators: Geophysics, 55, 306-319.

Hale, D., 1990, Stable explicit depth extrapulation of seismic wavefields: CWP-095, Colorado School of Mines.

Hazra, S. N., and Reddy, M. S., 1986, Design of circularly symmetric low-pass twodimensional FIR digital filters using transformation: IEEE Trans. Circuits Syst., CAS-33, 1022-1026.

Holberg, O., 1988, Towards optimum one-way wave propagation: Geophys. Prosp., $36,99-114$.

Kitchenside, P., and Jakubowicz, H., 1987, Operator design for 3-D depth migration: 57th Ann. Internat. Mtg., Soc. Expl. Geophys., Expanded Abstracts, 556-558.

Kitchenside, P., 1988, Steep dip 3-D migration: some issues and examples: 58th Ann. Internat. Mtg., Soc. Expl. Geophys., Expanded Abstracts, 076-978.

Kosloff, D., and Kessler, D., 1987, Accurate depth migration by a generalized phaseshift method: Geophysics, 52, 1074-1084.

McClellan, J. H., 1973, The design of two-dimensional digital filters by transformations: Proc. 7th Annual Princeton Conf. on Inform. Sci. and Syst., 247-251.

McClellan, J. H., and Chan, D. S., 1977, A 2-D FIR filter structure derived from the Chebyshev recursion: IEEE Trans. Circuits Syst., CAS-24, 372-378.

McClellan, J. H., and Parks, T. W., 1972, Equiripple approximation of fan filters: Geophysics, 37, 573-583.

Mecklenbräuker, W. G., and Mersereau, R. M., 1976, McClellan transformations for two-dimensional digital filtering: II -- implementation: IEEE Trans. Circuits Syst., CAS-23, 414-422.

Mersereau, R. M., Mecklenbräuker, W. G., and Quatieri, T. F., 1976, McClellan transformations for two-dimensiona! digital filtering: I - design: IEEE Trans. Circuits Syst., CAS-23, 405-414.

Reshef, M., and Kessler, D., 1989, Practical implementation of three-dimensional poststack depth migration: Geophysics, 54, 309-318.

Ristow, D., 1980, 3-D downward extrapolation of seismic data, in particular by finite difference methods: PhD thesis, University of Utrecht, The Netherlands.

Yilmaz, O., 1987, Seismic data processing: Soc. of Expl. Geophysics. 

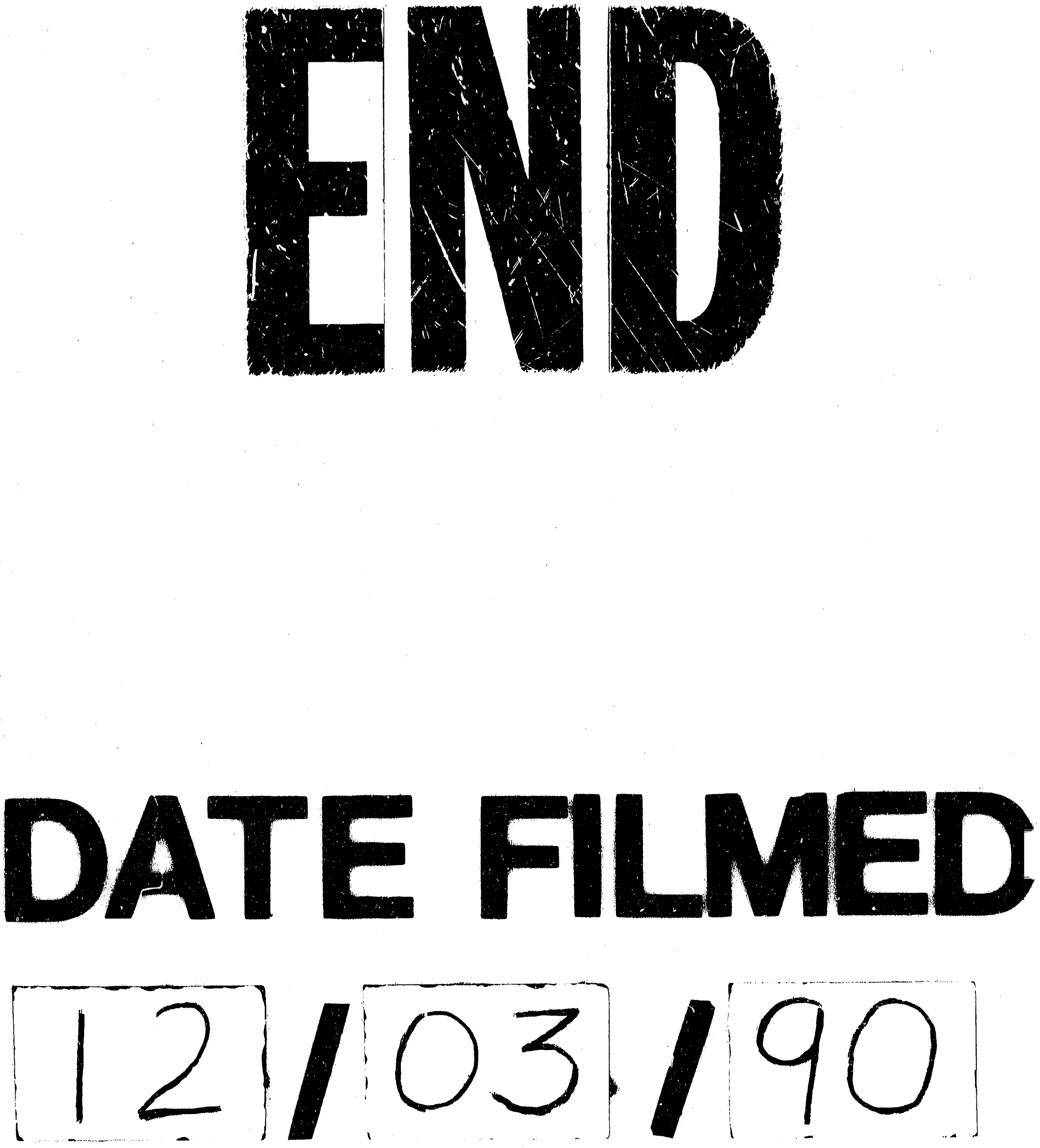
